\title{
Ultrasound-guided percutaneous drainage as an alternative to surgery in treating breast abscesses
}

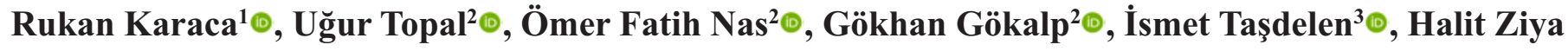 \\ Dündar ${ }^{3} \odot$
}

${ }^{1}$ Department of Radiology, Ceylanpınar State Hospital, Şanliurfa, Turkey

${ }^{2}$ Department of Radiology, Uludağ University School of Medicine, Bursa, Turkey

${ }^{3}$ Department of General Surgery, Uludă̆ University School of Medicine, Bursa, Turkey

DOI: $10.18621 /$ eurj.421325

\begin{abstract}
Objectives: The aim of this study was to assess whether ultrasound-guided (US-guided) percutaneous drainage of breast abscesses could be used as an alternative to surgery.

Methods: We performed a retrospective study. Twenty patients were included in the study who were diagnosed as having a non-specific breast abscess. Eleven patients underwent surgery whereas nine patients were treated with US-guided drainage. Ultrasonographic findings, results of treatment and follow-up were evaluated between the two groups.

Results: In the US-guided drainage group, 5 patients were treated with needle aspiration and 4 were treated with catheter drainage. All cases within the needle aspiration group totally recovered. However, one case within the catheter drainage group failed. The total success rate of US-guided drainage was $88.8 \%$. The median followup period was 21.0 days in the US-guided drainage group and 45.0 days in the surgical drainage group. There were no statistically significant differences in terms of recovery $(p=0.450)$ and follow-up periods $(p=0.112)$ between the surgical drainage and US-guided drainage groups.

Conclusions: US-guided percutaneous drainage may be preferred as a first method of choice in treatment of a breast abscess. The most appropriate approach to breast abscess treatment will be possible with a multidisciplinary approach of surgery and radiology.
\end{abstract}

Keywords: Breast abscess, percutaneous drainage, US-guided drainage

Received: May 5, 2018; Accepted: April 30, 2019; Published Online: June 30, 2019.

B reast abscesses often develop as a complication of infectious mastitis in young women [1]. Traditional treatment includes surgical drainage and systemic administration of antibiotics. Surgery, which usually requires general anesthesia, leads to scar tissue, also requires the cessation of breast-feeding during treatment and is more expensive. However, ultrasound-guided (US-guided) percutaneous drainage is a feasible, safe, well-tolerated and successful method [2-4].

In our study, we aimed to show that US-guided drainage of breast abscesses may be an alternative to surgical incision and drainage. 


\section{METHODS}

Written consent of the ethical committee of Uludağ University School of Medicine, dated February $10^{\text {th }}, 2015$ and numbered 2015-3/21, was received for this study planned retrospectively. We evaluated 9 patients who were diagnosed with breast abscess and underwent US-guided abscess drainage between December 2013-December 2014 and 11 patients who were diagnosed with breast abscess and underwent surgery between January 2009-December 2014. The patients who had a suspected breast abscess on ultrasound examination and were reported to have a non-specific infection in cutting needle biopsy (CNB) were included in the study. We evaluated clinical information, ultrasound images, antibiotic therapies, biopsy results, US-guided drainage method, microbiology and antibiotic susceptibility test results that were obtained from the files of the patients. The patients were divided into two groups, including those undergoing surgical drainage and those undergoing US-guided drainage. Ultrasound examinations were performed using a 7.5 MHz linear probe (Toshiba Medical Systems Corporation, Otawara, Tochigi, Japan) with a Xario device (Toshiba Medical Systems Corporation, Otawara, Tochigi, Japan).

The patients underwent CNB during percutaneous drainage to exclude conditions that imitate breast abscess. The procedure was performed under local anesthesia. CNB was performed using a fully automatic gun (Bard MAGNUM, Covington, GA) and a $14 \mathrm{G}$ needle with the freehand method under ultrasound. At least 2 samples were taken from each lesion, and the materials were sent for pathological examination in $10 \%$ formaldehyde solution.

\section{US-guided drainage}

US-guided drainage was performed under sterile conditions with local anesthesia. Abscesses smaller than $5 \mathrm{~cm}$ firstly underwent fine needle aspiration. 1820G one-wall needles (Seldinger) were used for aspiration. If abscess material was highly viscous, $18 \mathrm{G}$ needles were preferred. Catheter drainage was performed for abscesses larger than $5 \mathrm{~cm}$ and also for abscesses larger than $3 \mathrm{~cm}$ repeating within 7-14 days after fine needle aspiration. Catheter drainage was performed under ultrasound and fluoroscopy device (AXIOM Artis, Siemens, Erlangen, Germany).
Fluoroscopy was performed to determine the depth of the abscess cavity and its relation to the chest wall. The abscess cavity was entered using an $18 \mathrm{G}$ needle (Seldinger) under local anesthesia. The abscess cavity was then evaluated by giving contrast agent after about 1-2 cc of aspiration. After guidewire placement, a drainage catheter was placed over the guidewire. $6-8 \mathrm{~F}$ drainage catheters (Neo-Hydro, Bioteq, Taiwan) were used for drainage.

Saline lavage was also applied during drainage of the abscesses. Saline was injected with a $10 \mathrm{ml}$ syringe into the abscess and aspiration was performed until the aspirated content was cleaned.

Oral antibiotic treatment was given by the department of breast surgery for patients who underwent percutaneous drainage. The material obtained during the aspiration process was sent to microbiology for culture. Antibiotherapy was revised during the follow-up according to the antibiotic susceptibility profile.

A decrease in inflammatory symptoms such as fever, erythema and pain was evaluated as treatment response and good clinical response. Only clinical follow-up was done after drainage for abscesses showing a good clinical response. An ultrasound control was done once 14 days later to show that the abscess fully regressed. Ultrasound was repeated until complete regression occurred for patients with a partial clinical response. Controls were done at 7 or 14-day intervals. Control intervals were adjusted according to the clinical findings of the patients.

\section{Statistical Analysis}

Categorical data are summarized as number and percentage. Fisher's exact and chi-square test were used to compare the data. Numerical data were defined as median, minimum and maximum values. MannWhitney U-test was used to compare numerical data. The significance level was accepted as $p<0.05$ for all tests.

\section{RESULTS}

Demographic information, lesion characteristics and follow-up period of the patients included in the study are shown in Table 1. The age of the patients included in the study ranged from 20 to 63 and the 
Table 1. Distribution of lesions according to the form of drainage

\begin{tabular}{lcccc}
\hline & $\begin{array}{c}\text { US-guided drainage } \\
(\mathbf{n}=\mathbf{9})\end{array}$ & $\begin{array}{c}\text { Surgical drainage } \\
(\mathbf{n}=\mathbf{1 1})\end{array}$ & $\begin{array}{c}\text { Total } \\
(\mathbf{n}=\mathbf{2 0})\end{array}$ & $p$ value \\
\hline $\begin{array}{l}\text { Age (years) } \\
\text { (median (min-max)) }\end{array}$ & $29.0(20-63)$ & $33.0(26-54)$ & $33.0(20-63)$ & 0.603 \\
$\begin{array}{l}\text { Lactational status } \\
\text { (P/ NP) }\end{array}$ & $1 / 8$ & $0 / 11$ & $1 / 19$ & 0.450 \\
$\begin{array}{l}\text { Localization } \\
\text { (right/ left) }\end{array}$ & $5 / 4$ & $4 / 7$ & $9 / 11$ & 0.653 \\
$\begin{array}{l}\text { Size (mm) } \\
\text { (median (min-max)) }\end{array}$ & $40.0(16-80)$ & $20.0(10-80)$ & $30.5(10-80)$ & 0.261 \\
$\begin{array}{l}\text { Follow up (day) } \\
(\text { median (min-max)) }\end{array}$ & $21.0(7-80)$ & $45.0(18-65)$ & $37.5(7-80)$ & 0.112 \\
\hline
\end{tabular}

$\min =$ minimum, $\max =$ maximum, $\mathrm{P}=$ Puerperal, $\mathrm{NP}=$ Non-puerperal

median age was 33.0 years.

Of 20 abscesses included in the study, 1 (5\%) was within the puerperal period and $19(95 \%)$ were within the non-puerperal period. One patient with puerperal abscess was within the tenth month postpartum and underwent US-guided drainage (Figure 1). This patient was treated with US-guided needle aspiration. Of the patients with non-puerperal abscess, 8 (42.1\%) underwent US-guided drainage and 11 (57.9\%) underwent surgical drainage. One of the patients with non-puerperal abscess was 7 weeks pregnant. Of the non-puerperal abscesses that underwent US-guided drainage, $4(50 \%)$ were treated with needle aspiration and $4(50 \%)$ were treated with catheter drainage (Figure 2).

The patients were followed for periods ranging between 7-80 days. The median follow-up period was 21.0 days (7-80 days) in the group that underwent USguided drainage and 45.0 days (18-65 days) in the group that underwent surgical drainage.

When looking at the culture results of the abscesses that underwent US-guided drainage, Staphylococcus aureus (S. aureus) was isolated from one patient, Enterococcus faecalis was isolated from

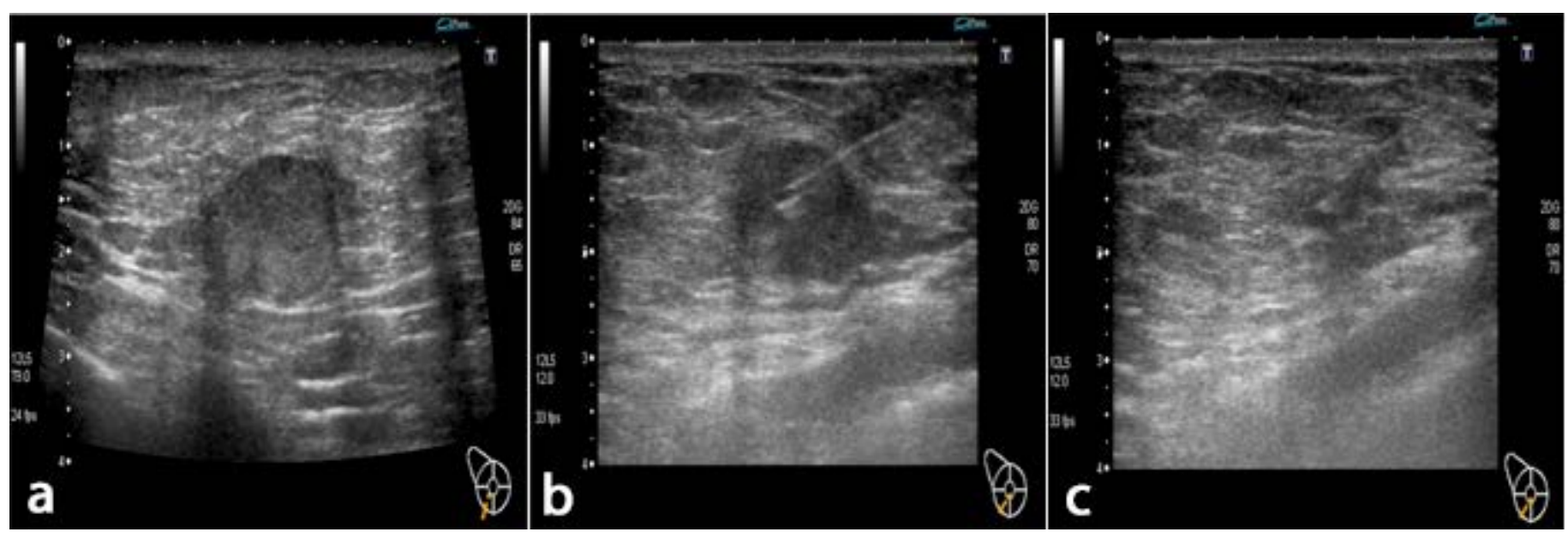

Figure 1. A 28-year-old puerperal female patient noticed tenderness and redness in the lower outer quadrant of the right breast during breastfeeding. (a) On ultrasound examination, a heterogeneous abscess was seen in the right breast at 7 o'clock position with a size of $18 \times 13 \mathrm{~mm}$. (b and c) Aspiration was performed with an 18G needle and CNB was performed with a $14 \mathrm{G}$ needle under ultrasound. The aspirated purulent material was sent for culture. The abscess disappeared on ultrasound examination made immediately after the aspiration. There was no growth in culture and the pathological result was reported as non-specific mastitis. 

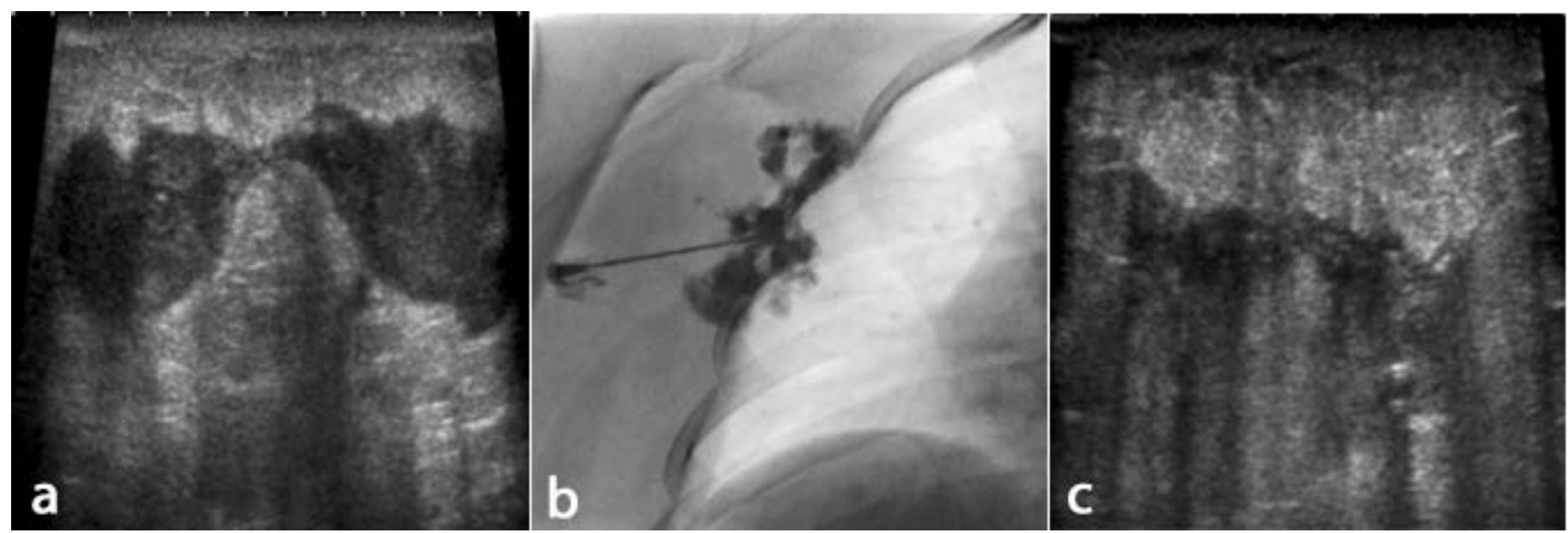

Figure 2. A 34-year-old non-puerperal female patient. She was admitted with ongoing pain and swelling in the right breast for three months. She had received antibiotherapy before she was admitted to our department. (a) Interconnected and content-heavy collections were found in a $61 \times 45 \mathrm{~mm}$ area in the right breast at 2 o'clock position on ultrasound examination. (b) She underwent catheter drainage and also the abscess was seen to be multilocular with contrast agent injected into the abscess cavity during the process. (c) $8 \mathrm{~F}$ drainage catheter was inserted into the abscess and $15 \mathrm{ml}$ of purulent material was aspirated. There was no growth in culture. The patient recovered after antibiotic therapy and drainage.

one patient and Escherichia coli was isolated from one patient. In the surgical drainage group, S. aureus was isolated from one patient, Actinomyces israelii was isolated from one patient and Mycobacterium tuberculosis was isolated from one patient. There was no growth in the cultures of the other patients.

In the non-puerperal group who underwent USguided drainage, $3(37.5 \%)$ of 8 patients presented with a recurrent abscess. There was no recurrent abscess in the puerperal group. The patients with a recurrent abscess underwent repeat aspiration. Of these patients, $2(66.6 \%)$ improved after the second aspiration and 1 underwent a third aspiration. In this patient, the subareolar abscess was seen on ultrasound examination done on the 7 th day after the third aspiration, and then this patient was referred to surgery. In this patient, who was reported to have a non-specific infection in CNB at the initial diagnosis, the postoperative pathology result changed to granulomatous lobular mastitis.

In our study, of 9 abscesses that underwent USguided drainage, $5(55.5 \%)$ underwent needle

Table 2. Characteristics and follow-up results of patients who underwent US-guided drainage

\begin{tabular}{ccccccccc}
\hline Age & $\begin{array}{c}\text { Lactation } \\
\text { status }\end{array}$ & $\begin{array}{c}\text { Localization } \\
\text { of abscess }\end{array}$ & $\begin{array}{c}\text { Structure } \\
\text { of abscess }\end{array}$ & $\begin{array}{c}\text { Size } \\
(\mathbf{m m})\end{array}$ & $\begin{array}{c}\text { Type of } \\
\text { treatment }\end{array}$ & $\begin{array}{c}\text { Number of } \\
\text { aspirations }\end{array}$ & $\begin{array}{c}\text { Pathogenic } \\
\text { organism }\end{array}$ & Follow up \\
\hline 29 & NP & Peripheral & Multilocular & $45 \times 22$ & Catheter & 3 & - & Surgery \\
26 & NP & Peripheral & Multilocular & $43 \times 20$ & Catheter & 2 & - & Resorption \\
28 & P & Peripheral & Unilocular & $18 \times 13$ & NA & 1 & - & Resorption \\
34 & NP & Peripheral & Multilocular & $61 \times 45$ & Catheter & 1 & - & Resorption \\
27 & NP & Peripheral & Multilocular & $37 \times 16$ & NA & 2 & - & Resorption \\
48 & NP & Peripheral & Multilocular & $26 \times 14$ & NA & 1 & S. aureus & Resorption \\
57 & NP & Central & Multilocular & $80 \times 50$ & Catheter & 1 & - & Resorption \\
63 & NP & Peripheral & Unilocular & $16 \times 9$ & NA & 1 & E. faecalis & Resorption \\
20 & NP & Central & Unilocular & $40 \times 20$ & NA & 1 & E. coli & Resorption \\
\hline
\end{tabular}

$P=$ Puerperal, NP $=$ Non-puerperal, NA $=$ Needle aspiration, S. aureus $=$ Staphylococcus aureus, E. faecalis $=$

Enterococcus faecalis, E. coli= Escherichia coli 
Table 3. Characteristics and follow-up results of patients who underwent surgical drainage

\begin{tabular}{lcccccc}
\hline Age & $\begin{array}{c}\text { Lactation } \\
\text { status }\end{array}$ & $\begin{array}{c}\text { Localization } \\
\text { of abscess }\end{array}$ & $\begin{array}{c}\text { Structure of } \\
\text { abscess }\end{array}$ & $\begin{array}{c}\text { Size } \\
(\mathbf{m m})\end{array}$ & $\begin{array}{c}\text { Pathogenic } \\
\text { organism }\end{array}$ & Follow up \\
\hline 33 & NP & Peripheral & Unilocular & $10 \times 8$ & - & Resorption \\
54 & NP & Central & Unilocular & $20 \times 20$ & A. israelii & Resorption \\
35 & NP & Central & Unilocular & $15 \times 13$ & S. aureus & Resorption \\
42 & NP & Central & Multilocular & $50 \times 50$ & - & Resorption \\
29 & NP & Peripheral & Multilocular & $60 \times 40$ & - & Resorption \\
39 & NP & Central & Unilocular & $20 \times 15$ & - & Resorption \\
26 & NP & Peripheral & Unilocular & $35 \times 20$ & M.tuberculosis & Resorption \\
30 & NP & Peripheral & Unilocular & $17 \times 8$ & - & Resorption \\
33 & NP & Central & Multilocular & $80 \times 10$ & - & Resorption \\
32 & NP & Peripheral & Multilocular & $20 \times 12$ & - & Resorption \\
34 & NP & Central & Multilocular & $20 \times 20$ & - & Resorption \\
\hline
\end{tabular}

$\mathrm{NP}=$ Non-puerperal, A. israelii $=$ Actinomyces israelii, S. aureus $=$ Staphylococcus aureus, M. tuberculosis $=$ Mycobacterium tuberculosis

aspiration and $4(44.5 \%)$ underwent catheter drainage. All patients who underwent needle aspiration improved (100\% success rate). Of the patients who underwent catheter drainage, 1 did not improve and was sent to surgery ( $75 \%$ success rate). The total success rate of US-guided drainage was calculated as $88.8 \%$. Compared with the surgical group, there was no statistically significant difference between the two groups in terms of recovery rate $(p=0.450)$.

The data and follow-up results of the US-guided drainage group and surgical drainage group are shown in detail in Tables 2 and 3.

\section{DISCUSSION}

The traditional treatment of breast abscesses has been considered to be surgical drainage and systemic antibiotics until the 1990s. In 1990, Karstrup et al. [3] showed that US-guided drainage can be applied as an alternative to surgery. In light of many studies, today US-guided drainage combined with oral antibiotic therapy is used as an effective treatment for breast abscesses. US-guided percutaneous drainage is an advantageous method in many ways in terms of the patient. It does not require general anesthesia and there is no need for hospitalization and postoperative care. It is a minimally invasive method and can be performed in a short time under outpatient conditions. The other advantages of this method may be that it results in minimal or no scar tissue, does not require stopping breastfeeding, is cheaper than a surgical approach and has similar or lower complication rates compared to surgery. However, it has disadvantages such as the formation of cutaneous fistula after percutaneous drainage and a repeating collection, especially in puerperal patients.

The success of percutaneous drainage varies in a wide range of 54-100\% [5-9]. The large difference between the percentage of successes may be due to many factors such as the type of abscess, the size of abscess, the technique used, using antibiotic therapy along with drainage and the success criteria selected.

Different techniques can be used in percutaneous abscess drainage. Ultrasound is usually preferred as a guide method. However, drainage was performed with direct palpation without using ultrasound in some studies [10, 11]. US-guided drainage facilitates entering especially small and multilocular abscesses and performing adequate drainage. All percutaneous interventions in our study were conducted under ultrasound. In addition to ultrasound, the abscess cavity was evaluated by giving contrast agent under fluoroscopy to determine the depth of the abscess cavity and its relation to the chest wall in patients who underwent catheter drainage. 
The thickness of the needle used for aspiration can influence success. In reported studies, $14-25 \mathrm{G}$ needles were usually used $[5,6,8,12]$. The choice of needle thickness varies according to the content of the abscess. For example, a dark and viscous abscess is more easily aspirated with a thick needle. However, the use of a thick needle may be a risk factor for development of a cutaneous fistula. Moreover, a thick needle can lead to pain in the sensitive breast due to inflammation, despite local anesthesia. In our study, 18 and $20 \mathrm{G}$ needles were preferred. If the abscess material was highly viscous, $18 \mathrm{G}$ needles provided sufficient aspiration. Because of the small number of patients in our study, there was no comment on changes in the success rate with needle thickness.

Aspiration may not always be sufficient in percutaneous drainage of large collections and abscesses. In some studies in the literature, catheter drainage was preferred instead of needle aspiration in the treatment of large collections and abscesses larger than $3 \mathrm{~cm}[6,8,13]$. In our study, at the time of diagnosis, abscesses larger than $5 \mathrm{~cm}$ underwent catheter drainage and abscesses smaller than $5 \mathrm{~cm}$ underwent fine needle aspiration. Abscesses that repeated within 7-14 days after fine needle aspiration during the follow-up and were larger than $3 \mathrm{~cm}$ underwent catheter drainage. It is an advantage that the catheter remains within the cavity until the abscess is completely drained. Thus, it is not necessary to enter with the needle again and again. However, the catheter remaining for a long time can also cause cutaneous fistula and it can be a source of infection. The presence of the catheter within the breast may affect patient comfort. The catheter should be taken out as early as possible. In the studies in the literature, the residence time of the catheter varies. For example, in the study of Ulitzsch et al. [8], the residence time of the catheter within the breast ranged from 1 to 25 days and the mean duration was 6.4 days. In the study of Christensen et al. [6], in which a drainage catheter was performed in puerperal and non-puerperal abscesses, the residence time of the catheter within the breast ranged from 2 to 6 days and the median residence time was stated as 4 days. In our study, the median residence time was 8 days (between 6-10 days) in abscesses. The median residence time was longer in our study compared to other studies in the literature due to the small number of patients. The catheter stayed for 10 days in one patient and also it was changed on the 7 th day in this patient. This patient decreased the homogeneity of the residence time due to the small number of patients.

Recent studies have shown that breast abscesses can be treated by repeated needle aspiration without the use of a catheter $[7,8,12]$. In a case series of 26 patients, Imperial et al. [7] stated that the success rate was $96 \%$. In a case series of 39 patients, Elagili et al. [12] stated that the success rate was $83.3 \%$. In our study, $5(55.5 \%)$ of 9 abscesses that underwent USguided drainage underwent needle aspiration. All patients who underwent needle aspiration improved and the success rate was $100 \%$ in these patients. An $80 \%$ decrease was achieved after the first aspiration and complete regression (100\%) was achieved after the second aspiration. They were treated with percutaneous drainage without the need for surgery.

Systemic antibiotics should always be given at any time in addition to percutaneous drainage in the treatment of breast abscess. Antibiotherapy is regulated according to the most common agent. Although the material that was taken for determining the appropriate antibiotic was sent for culture, there was no growth in the culture because antibiotherapy had been initiated. In the literature, Ozseker et al. [2] and Imperiale et al. [7] reported that sterile culture results were $36 \%$ and $23 \%$, respectively. Many studies have shown that the most common organism obtained in culture was S. aureus $[14,15]$. In our study, there was no growth in a significant part $(66.6 \%)$ of the culture results. This rate was higher compared to the other studies in the literature and this is due to the high use of antibiotics before drainage.

A breast abscess is a disease whose treatment is difficult. Surgical treatment, as well as percutaneous treatment, may fail. After US-guided percutaneous drainage, patients are recommended to surgery if several attempts (at least 3-5) fail but this decision may also vary depending on the clinical condition of the patient [1]. In a case series of 13 patients, Hook and Ikeda [5] reported that the recovery rate was $54 \%$. In this study, treatment failure was seen in abscesses larger than $3 \mathrm{~cm}$ or subareolar abscesses. In a case series of 26 patients, Imperiale et al. [7] stated that one patient with a large and subareolar abscess did not improve and this patient was directed to surgery. In our study, only one patient (1 of 9 patients) failed and 
also this patient was directed to surgery because recurrence occurred after the third attempt.

\section{Limitations}

Our study had some limitations. The first limitation of our study was the retrospective design. Our study had a limited number of patients, and our results should be verified with a larger series.

\section{CONCLUSION}

In conclusion, as shown in previous studies, our study confirms with a success rate of $88.8 \%$ that USguided percutaneous drainage can be used as an alternative treatment to surgery. US-guided percutaneous drainage combined with oral antibiotics should be preferred as the primary treatment in the treatment of breast abscesses, and also a surgical approach should be performed for recurrent abscesses in which percutaneous drainage has failed. Radiologists should play a role in the treatment as well as in the diagnosis of breast abscesses, and also the management of breast abscesses should be performed by a multidisciplinary team with a surgeon.

\section{Authorship declaration}

All authors listed meet the authorship criteria according to the latest guidelines of the International Committee of Medical Journal Editors, and all authors are in agreement with the manuscript.

\section{Conflict of interest}

The author disclosed no conflict of interest during the preparation or publication of this manuscript.

\section{Financing \\ The author disclosed that they did not receive any grant during conduction or writing of this study.}

\section{REFERENCES}

[1] Trop I, Dugas A, David J, El Khoury M, Boileau JF, Larouche $\mathrm{N}$, et al. Breast abscesses: evidence-based algorithms for diagnosis, management and follow-up. Radiographics 2011;31:1683-99.

[2] Ozseker B, Ozcan UA, Rasa K, Cizmeli OM. Treatment of breast abscesses with ultrasound-guided aspiration and irrigation in the emergency setting. Emerg Radiol 2008;15:105-8.

[3] Karstrup S, Nolsoe C, Brabrand K, Nielsen KR. Ultrasonically guided percutaneous drainage of breast abscesses. Acta Radiol 1990;31:157-9.

[4] Boakes E, Woods A, Johnson N, Kadoglou N. Breast infection: a review of diagnosis and management practices. Eur J Breast Health 2018;14:136-43.

[5] Hook GW, Ikeda DM. Treatment of breast abscesses with USguided percutaneous needle drainage without indwelling catheter placement. Radiology 1999; 213:579-82.

[6] Christensen AF, Al-Suliman N, Nielsen KR, Vejborg I, Severinsen N, Christensen H, et al. Ultrasound-guided drainage of breast abscesses: results in 151 patients. $\mathrm{Br} \mathrm{J}$ Radiol 2005;78:186-8.

[7] Imperiale A, Zandrino F, Calabrese M, Parodi G, Massa T. Abscesses of the breast: US-guided serial percutaneous aspiration and local antibiotic therapy after unsuccessful systemic antibiotic therapy. Acta Radiol 2001;42:161-5.

[8] Ulitzsch D, Nyman MK, Carlson RA. Breast abscess in lactating women: US-guided treatment. Radiology 2004;232:904-9.

[9] Bushra S, Azhar AS, Khush MS, Roshan AS. Needle aspiration in treatment of breast abscess. RMJ 2014;39:58-60.

[10] Schwarz RJ, Shrestha R. Needle aspiration of breast abscesses. Am J Surg 2001;182:117-9.

[11] Tan SM, Low SC. Non-operative treatment of breast abscesses. Aust N Z J Surg 1998;68:423-4.

[12] Elagili F, Abdullah N, Fong L, Pei T. Aspiration of breast abscess under ultrasound guidance: outcome obtained and factors affecting success. Asian J Surg 2007;30:40-4.

[13] Berna-Serna JD, Madrigal M, Berna-Serna JD. Percutaneous management of breast abscesses: an experience of 39 cases. Ultrasound Med Biol 2004;30:1-6.

[14] Munakomi S, Adhikary S. Clinical profile and methodology in the management of inflammatory benign breast lesions. Cureus 2014;6:e214.

[15] Ranjeesh V, Kotha S. A prospective comparative study of needle aspiration vs incision and drainage of lactational breast abscess. IJCMR 2018;5:E13-7. 